\title{
Mortality predictors after percutaneous coronary intervention - a prospective single-center registry study
}

László Hadadi', Paul Calburean',2, Paul Grebenisan', Victor Vacariu', Reka-Katalin Drincal', Oana Tepes', lulia Grancea', loana Sus',2, Cristina Somkereki',2, Valentin Simon', Zoltán Demjén', István Adorján', Irina Pinitilie', Anca Teodora Dolcos', Tiberiu Oltean'

\begin{abstract}
Objectives - To evaluate the predictors of three-year cardiovascular mortality after percutaneous coronary intervention (PCl) in a Romanian tertiary cardiovascular center. Methods - Consecutive patients treated by $\mathrm{PCl}$ in the Emergency Institute for Cardiovascular Diseases and Transplantation of Targu Mures were included prospectively in a local $\mathrm{PCl}$ Registry. Demographic, clinical, and procedural parameters of the patients enrolled in the year 2016 were statistically analyzed as possible predictors of three-year cardiovascular mortality post-PCI. Results - 1079 patients were included: 254 (23.5\%) with ST-segment elevation acute myocardial infarction (STEMI), 278 (25.8\%) with non-ST segment elevation acute coronary syndrome (NSTEACS) and 547 (50.7\%) with chronic coronary syndrome (CCS). Three-year cardiovascular mortality was $20.1 \%, 10.8 \%$ and $5.7 \%$ after PCI for STEMI, NSTEACS and CCS, respectively. Cox proportional hazards regression evidenced as independent predictors of long-term mortality after PCl: low left ventricular ejection fraction (LVEF), renal dysfunction, presentation with cardiogenic shock or with cardiac arrest in the case of acute coronary syndromes, and the history of significant valvular heart disease and low LVEF in the case of CCS (all p $\leq 0.0$ I). Conclusions - Simple clinical variables but no procedural factors were the main predictors of 3-year cardiovascular mortality after $\mathrm{PCl}$ in this all-comers population.
\end{abstract}

Keywords: coronary artery disease, percutaneous coronary intervention, mortality.

Rezumat: Objective - Evaluarea predictorilor mortalității cardiovasculare pe termen lung după intervențiile coronariene percutane ( $\mathrm{PCl}$ ) efectuate într-un centru cardiovascular terțiar român. Metode - Pacienți consecutivi tratați prin $\mathrm{PCl}$ au fost incluși prospectiv în Registrul PCl al Institutului de Urgență pentru Boli Cardiovasculare și Transplant din Târgu Mureș. Datele demografice, clinice și procedurale ale pacienților din 2016 au fost analizate statistic ca predictori posibili ai mortalității cardiovasculare la trei ani post-PCI. Rezultate - Au fost incluși 1079 pacienți: 254 (23,5\%) cu infarct miocardic acut cu supradenivelare de segment-ST (STEMI), $278(25,8 \%)$ cu sindrom coronarian acut fară supradenivelare de segment-ST (NSTEACS) si $547(50,7 \%)$ cu sindrom coronarian cronic (CCS). Mortalitatea de cauză cardiovasculară la 3 ani post-PCl s-a ridicat la: 20,1\% după STEMI, 10,8\% după NSTEACS și 5,7\% după CCS. Analiza de regresie multivariabilă Cox a evidențiat ca predictori independenți ai mortalității pe termen lung: fracția de ejecție a ventriculului stâng (LVEF) deprimată, disfuncția renală, prezentarea cu șoc cardiogen sau post-resuscitare în cazul sindroamelor coronariene acute; LVEF scăzută și prezența bolii valvulare semnificative în cazul CCS (toate valorile $p \leq 0,0$ I). Concluzii - Variabile clinice simple, dar nu și cele procedurale au fost asociate în mod independent cu mortalitatea pe termen lung în populația analizată.

Cuvinte cheie: boală coronariană, intervenție coronariană percutană, mortalitate.

\section{BACKGROUND}

The diseases of the circulatory system are the leading cause of death in the European Union, accounting for $37 \%$ of the total mortality in this territory'. Cardiovascular mortality is even higher in Romania: according to the European health statistics, $58.2 \%$ of all deaths had

\footnotetext{
' Emergency Institute for Cardiovascular Diseases and Transplantation, Targu Mures, Romania

2 „George Emil Palade” University of Medicine, Pharmacy, Science and

Technology, Targu Mures, Romania
}

a cardiovascular cause in 2016 in our country². The main pathology responsible for this high mortality rate is coronary artery disease (CAD), with standardized death rates rising to 368.4 and 247.5 per 100000 males and females respectively ${ }^{2}$. Percutaneous coronary intervention $(\mathrm{PCl})$ is the most frequently performed 
cardiovascular procedure in Europe ${ }^{2}$. Although in Romania there was a $146 \%$ increase in the number of PCls between 2012 and 20I7, from 82.7 to 120.7 procedures per 100000 residents, the country remained in the upper quartile of the European Union regarding CAD mortality in $2016^{2}$.

The short-term clinical evolution of patients treated by $\mathrm{PCl}$ in Romania is well documented, most of the studies reporting in-hospital- or 30-day mortality as a clinical outcome $\mathrm{e}^{3-11}$. However, contemporary data regarding long term mortality of CAD treated by $\mathrm{PCl}$ is mainly lacking in Romania: a few studies evaluated this subject, and only for special $\mathrm{PCl}$ indications, such as acute ST-elevation myocardial infarction (STEMI; I-year follow-up) $)^{12,13}$ or STEMI complicating left main CAD (I- and 3-year follow-up) ${ }^{14,15}$.

The present study analyzes the predictors of 3-year cardiovascular mortality in an all-comers patient population treated by $\mathrm{PCl}$ in a Romanian tertiary cardiovascular center.

\section{MATERIAL AND METHODS}

\section{Study population}

All patients older than 18 years and treated by $\mathrm{PCl}$ in the Emergency Institute for Cardiovascular Diseases and Transplantation of Târgu Mureș have been included prospectively after hospital discharge in the local PCI Registry of the Institute since January 0I. 2016. The Registry is accessible on-line at the website http:// pci.cardio.ro/, and is based on the criteria of Cardiology Audit and Registration Data Standards (CARDS) developed by the Department of Health and Children, European Society of Cardiology, Irish Cardiac Society, and the European Commission ${ }^{16}$. All the information available regarding all the variables proposed in that document were collected in case of each included patient, at every $\mathrm{PCl}$. Briefly, the CARDS recommendations address data regarding demographics, relevant medical history and comorbid conditions, clinical status at hospital admission, $\mathrm{PCl}$ indication, affected and instrumented coronary artery segments, different invasive diagnostic and therapeutic devices, procedural complications, medical treatment and in-hospital evolution ${ }^{16}$. In the current analysis were included consecutive patients treated by $\mathrm{PCl}$ for an acute- or chronic coronary syndrome during the year 2016, as 3-year mortality data was available for this population. Acute coronary syndromes (ACSs) included STEMI (according to the universal definition of acute myocardial infarction ${ }^{17}$ ) and non-ST segment elevation acute coronary syndro- me (NSTEACS: unstable angina pectoris and acute myocardial infarction without persistent ST segment elevation, according to the definitions stated in the 2015 Guidelines of the European Society of Cardiology $\mathrm{ESC}^{18}$ ). All the other $\mathrm{PCl}$ procedures were performed electively in patients with stable angina pectoris and/ or documented myocardial ischemia. This latter group was recently redefined as chronic coronary syndromes $(\mathrm{CCS})^{19}$. Patients with missing data regarding any of the studied clinical/angiographic/follow-up parameters were excluded. However, very high-risk patients often excluded from clinical trials, such as those with cardiogenic shock or resuscitated cardiac arrest, were all included in the present analysis. Subjects with multiple interventions during 2016 were included only once, considering for analysis only the first $\mathrm{PCl}$ procedure. All patients (or their legal representatives) signed a written informed consent regarding their participation in the study. The study protocol complied with the Declaration of Helsinki and was approved by the Local Ethical Committee.

\section{Percutaneous coronary intervention}

All the $\mathrm{PCl}$ procedures were indicated and performed according to the clinical practice guidelines of the ESC considered current in $2016^{18,20}$. All patients received unfractionated heparin as periprocedural anticoagulation. The decision regarding the type of the implanted stent was left at the discretion of the operator in case of STEMI. In complex cases a heart-team based approach was used for decision-making regarding the best revascularization strategy. Like in case of other similar analyses, the procedure was considered successful when a final Thrombolysis in Myocardial Infarction (TIMI) flow $\geq 2$ was achieved with a residual stenosis of $<20 \%$ after stent implantation or $<50 \%$ after balloon angioplasty ${ }^{2 !}$.

\section{Assessed demographic, clinical, and procedural variables}

The following demographic and baseline clinical parameters were evaluated as possible predictors of mortality after PCl: patients' age and sex, the presence of cardiovascular risk factors (hypertension, diabetes mellitus, active smoking, hypercholesterolemia and obesity, i.e. body mass index $\geq 30 \mathrm{~kg} / \mathrm{m}^{2}$ ), medical history of: previous myocardial infarction, congestive heart failure, stroke, hemodynamically significant valvular heart disease or prior valvular heart surgery/replacement/intervention, peripheral vascular disease, previous $\mathrm{PCl}$ or coronary artery by-pass grafting. Additional clinical parameters assessed were: the left ventricular 

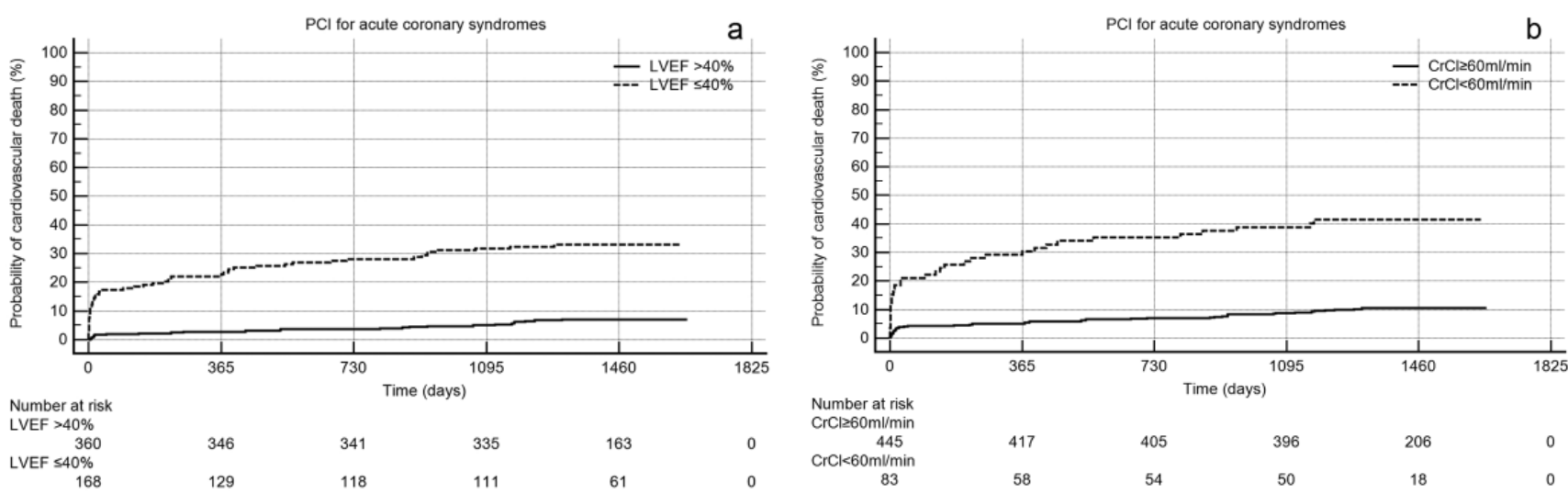

$$
\begin{array}{r}
\mathrm{CrCl} 260 \mathrm{~m} / / \mathrm{min} \\
445 \\
\mathrm{CrCl} 60 \mathrm{~m} / \mathrm{min} \\
83
\end{array}
$$

58

54

50
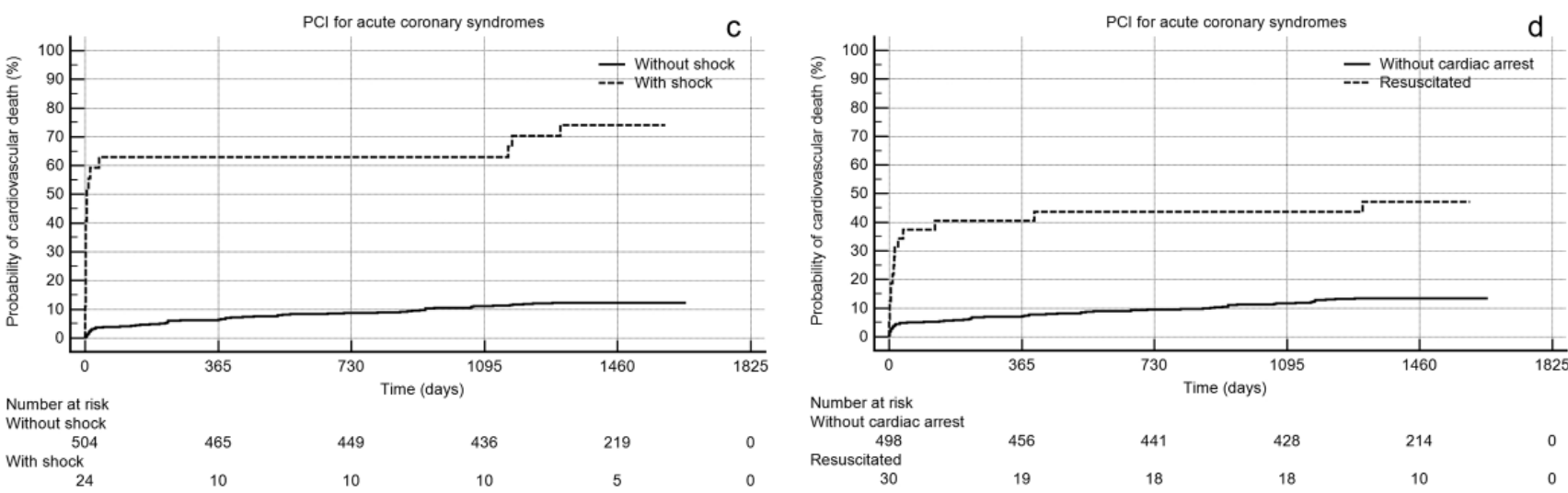

Figure I. Kaplan-Meier survival curves of acute coronary syndrome patients treated interventionally, according to different independent predictors of 3-year mortality: in case of depressed left ventricular systolic function (a), in the presence of renal dysfunction (b), in case of presentation with cardiogenic shock (c), or resuscitated cardiac arrest (d). All log-rank $\mathrm{p}$ values were $<0.00 \mathrm{I}$. $\mathrm{CrCl}$ - creatinine clearance, $\mathrm{LVEF}-$ left ventricular ejection fraction, $\mathrm{PCl}-$ percutaneous coronary intervention.
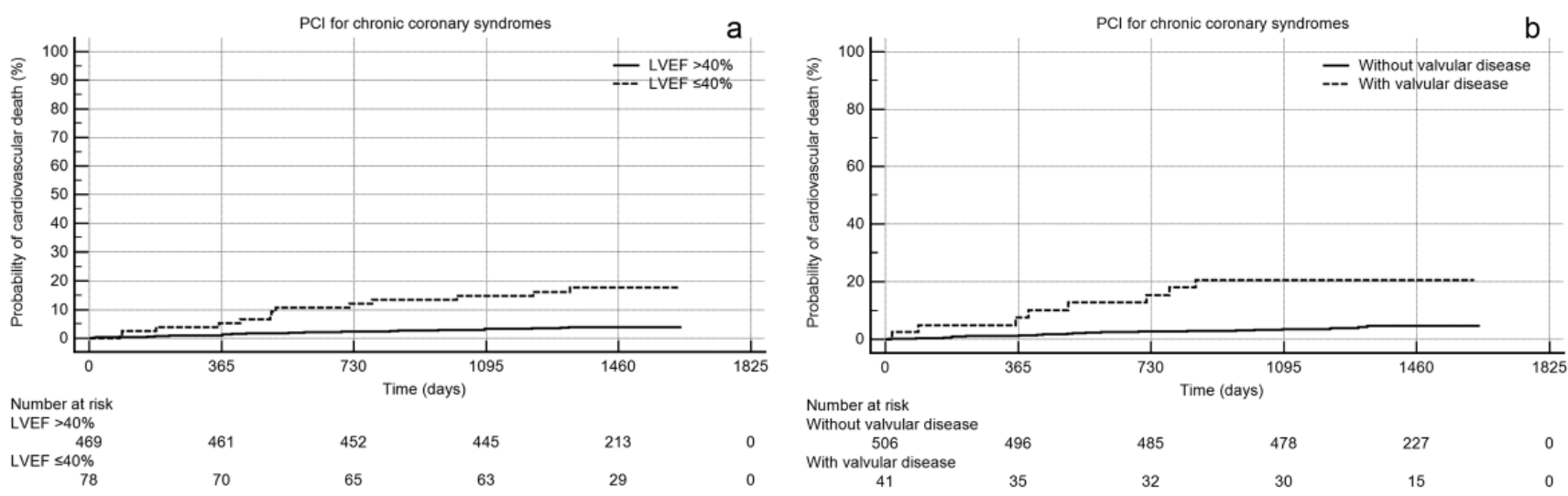

Figure 2. Kaplan-Meier survival curves of patients treated electively by $\mathrm{PCl}$, according to different independent predictors of 3-year mortality: in case of depressed left ventricular systolic function (a), and in the presence of hemodynamically significant valvular heart disease or prior valvular heart surgery/ replacement/intervention (b). All log-rank $\mathrm{p}$ values were $<0.00 \mathrm{I}$. LVEF - left ventricular ejection fraction, $\mathrm{PCl}-$ percutaneous coronary intervention. 
ejection fraction as determined by transthoracic echocardiography (the lowest value in percentage, during hospitalization), the presence of renal dysfunction (creatinine clearance $<60 \mathrm{ml} / \mathrm{min}^{21}$ as calculated according to the Cockroft-Gault formula ${ }^{22}$ ), presentation with cardiogenic shock or resuscitated cardiac arrest.

Regarding the $\mathrm{PCl}$ procedure, the subsequent variables were analyzed: the arterial access site (radial vs. other), the presence of triple-vessel disease (defined by the presence of at least one $\geq 50 \%$ diameter stenosis of a coronary branch with a diameter of $\geq 1.5 \mathrm{~mm}$ in the territory of the left anterior descending-, the left circumflex- and the right coronary artery or the presence of a stent in these vessels), left main stem disease (diameter stenosis $\geq 50 \%$ or the presence of a stent), TIMI flow after the $\mathrm{PCl}$, and the number and type (drug eluting vs. bare metal or a combination of these two) of the implanted stents. The occurrence of the following PCl-related complications was evaluated: acute vessel closure, coronary perforation, angiographic no-reflow phenomenon (TIMI flow $\leq 2$ observed during or at the end of $\mathrm{PCl}$, in the absence of dissection, residual stenosis, spasm or thrombus ${ }^{23}$ ), heart block requiring emergent pacing, resuscitation during $\mathrm{PCl}$, shock induced by the procedure, stroke, and post-procedural in-hospital major bleeding: overt clinical bleeding associated with a drop in $\mathrm{Hgb}$ of $>5 \mathrm{~g} / \mathrm{dl}(0.5 \mathrm{~g} / \mathrm{l})$ or in $\mathrm{Htc}$ of $15 \%{ }^{15}$. Data regarding the antiplatelet medication (aspirin, clopidogrel, ticagrelor and eptifibatide - the only glycoprotein IIB/IIIA blocker used) administered in the peri-procedural period was also analyzed.

\section{Clinical endpoint and follow-up}

The clinical endpoint of the present study was the incidence of cardiovascular death in the first 3 years of evolution after the index PCl. In-hospital mortality data was available from the $\mathrm{PCl}$ Registry. While I-, 2- and 3-year mortality rates were achieved from the database of the Romanian National Health Insurance System, the information regarding the exact date and cause (cardiovascular vs. other) of death were obtained from the Regional Statistics Office of the Romanian National Institute of Statistics.

\section{Statistical analysis}

To reduce the possibility of bias, the in-hospital, I-, 2- and 3-year death rates were statistically compared between the groups of included and excluded patients. Patients were categorized in three groups, according to $\mathrm{PCl}$ indication: those with STEMI, NSTEACS and CCS, respectively. The univariate predictors of 3-year cardiovascular mortality were separately assessed in these groups. Normal data distribution was tested with the help of the Kolmogorov-Smirnov test. Categorical variables were presented as frequencies (\%) and were analyzed using the chi-squared test. Continuous variables were summarized as medians (range) and were compared using the Mann-Whitney test or the Kruskal-Wallis test with Conover post-hoc analysis, as appropriate. Kaplan-Meier analysis with log-rank test was used to compare the survival curves of different patient groups. Two Cox multivariate proportional hazard models were built to identify the independent predictors of long-term cardiovascular mortality after $\mathrm{PCl}$ performed in patients with acute- and chronic coronary syndromes, respectively. In the first model were included all the variables presenting a statistically significant association with 3-year cardiovascular mortality at univariate analyses in patients with STEMI or NSTEACS. The second model contained the univariate mortality predictors after $\mathrm{PCl}$ performed for CCS. The following cut-off values were used for the continuous variables included in the Cox proportional hazard model: a $\mathrm{CrCl}$ of $<60 \mathrm{ml} / \mathrm{min}$ (a widely accepted threshold for the definition of chronic kidney disease ${ }^{21}$ ), a LVEF $\leq 40 \%$ (corresponding to an at least moderately depressed left ventricular systolic function ${ }^{24}$ ) and an age of $>62$ years; this latter was established by receiver operating characteristic (ROC) curve analysis. All tests were two-tailed, and a probability value of $<0.05$ was considered statistically significant. The statistical analysis was performed using the MedCalc Statistical Software version 19.4.I (MedCalc Software Ltd, Ostend, Belgium; https://www.medcalc. org; 2020).

\section{RESULTS}

\section{Study population}

In the PCl Registry of our institution were included II 68 patients in 2016. Eighty-nine of them were excluded because of missing clinical (52 patients), angiographic (33 patients) or follow-up (4 patients) data. In consequence, 1079 patients were included in the final statistical analysis. Their demographic, clinical and invasive procedural characteristics are presented according to the $\mathrm{PCl}$ indication in Table I. $\mathrm{PCl}$ was performed for a STEMI indication in 254 patients $(23.5 \%$ of the whole population) and for NSTEACS in 278 subjects ( $25.8 \%$ of the whole population). Procedural success was achieved in $96.8 \%$ of the included patients. More than $93 \%$ of the implanted stents were drug-elu- 


\begin{tabular}{|c|c|c|c|c|c|}
\hline \multirow{2}{*}{ Variable } & \multirow{2}{*}{$\begin{array}{c}\text { Total patients } \\
(\mathrm{N}=1079)\end{array}$} & \multicolumn{4}{|c|}{$\mathrm{PCl}$ indication } \\
\hline & & STEMI $(\mathrm{N}=254)$ & NSTEACS ( $\mathrm{N}=\mathbf{2 7 8})$ & $\operatorname{Ccs}(\mathrm{N}=547)$ & $\mathbf{p}$ \\
\hline Age (years) & $63.9(29-89)$ & $63.0(31-89)$ & $64.8(29-87)$ & $63.8(37-88)$ & 0.08 \\
\hline Female gender & $329(30.5)$ & $86(33.9)$ & $103(37.1)$ & $140(25.6)$ & 0.001 \\
\hline BMI $\left(\mathrm{kg} / \mathrm{m}^{2}\right)$ & $28.4(\mid 5.8-62.0)$ & $27.8(15.8-62.0)$ & $28.4(19.0-54.2)$ & $28.5(19.0-50.8)$ & 0.18 \\
\hline Arterial hypertension & $868(80.4)$ & $171(67.3)$ & $226(81.3)$ & $47 I(86.1)$ & $<0.001$ \\
\hline Diabetes mellitus & $335(31)$ & $65(25.6)$ & $95(34.2)$ & $175(32.0)$ & 0.08 \\
\hline Hypercholesterolemia & $640(59.3)$ & $110(43.3)$ & $165(59.4)$ & $365(66.7)$ & $<0.001$ \\
\hline Active smoking & $236(21.9)$ & $109(42.9)$ & $57(20.5)$ & $70(12.8)$ & $<0.001$ \\
\hline History of congestive heart failure & $15 \mid(14)$ & $27(10.6)$ & $45(16.2)$ & $79(14.4)$ & 0.16 \\
\hline History of valvular heart disease & $86(8.0)$ & $17(6.7)$ & $28(10.1)$ & $4 I(7.5)$ & 0.30 \\
\hline History of peripheral arterial disease & $146(13.5)$ & $13(5.1)$ & $35(12.6)$ & $98(17.9)$ & $<0.001$ \\
\hline History of stroke & $68(6.3)$ & $17(6.7)$ & $24(8.6)$ & $27(4.9)$ & 0.11 \\
\hline Previous myocardial infarction & $308(28.5)$ & $35(13.8)$ & $74(26.6)$ & $199(36.4)$ & $<0.001$ \\
\hline Previous $\mathrm{PCl}$ & $292(27.1)$ & $20(7.9)$ & $67(24.1)$ & $205(37.5)$ & $<0.001$ \\
\hline Previous CABG & $45(4.2)$ & $2(0.8)$ & II (4.0) & $32(5.9)$ & $<0.01$ \\
\hline $\mathrm{CrCl}(\mathrm{ml} / \mathrm{min})$ & $103.9(7.0-7 \mid 1.8)$ & $100.1(8.2-263.6)$ & $101.0(26.0-285.4)$ & $107.4(7.0-7|| .6)^{\#}$ & 0.01 \\
\hline Left ventricular ejection fraction (\%) & $50(10-80)$ & $45(10-60)^{\#}$ & $50(20-65)^{\#}$ & $55(20-80)^{\#}$ & $<0.001$ \\
\hline Presentation with cardiogenic shock & $27(2.5 \%)$ & $25(9.8)$ & $2(0.7)$ & $0(0.0)$ & $<0.001$ \\
\hline Cardiac arrest before $\mathrm{PCl}$ & $32(3.0)$ & $28(11.0)$ & $4(1.4)$ & $0(0.0)$ & $<0.001$ \\
\hline Radial arterial access site & $708(65.6)$ & $136(53.5)$ & $182(65.5)$ & $390(7 I .3)$ & $<0.001$ \\
\hline Three-vessel disease & $387(35.9)$ & $77(30.3)$ & $106(38.1)$ & $204(37.3)$ & 0.10 \\
\hline Left main stem disease & $88(8.2)$ & $16(6.3)$ & $29(10.4)$ & $43(7.9)$ & 0.20 \\
\hline TIMI flow<2 post-PCI & $35(3.2)$ & $6(2.4)$ & II (4.0) & $18(3.3)$ & $<0.58$ \\
\hline $\mathrm{PCl}$ without stent implantation & $81(7.5)$ & $19(7.5)$ & $26(9.4)$ & $36(6.6)$ & 0.36 \\
\hline Number of implanted stents $>1$ & $328(30.4)$ & $58(22.8)$ & $97(34.9)$ & $173(31.6)$ & $<0.01$ \\
\hline Only drug-eluting stents implanted* & $934(93.6)$ & $209(88.9)$ & $24 I(95.6)$ & $484(94.7)$ & $<0.01$ \\
\hline Dual antiplatelet therapy & $1031(95.6)$ & $248(97.6)$ & $267(96.0)$ & $516(94.3)$ & 0.09 \\
\hline $\begin{array}{l}\text { Clopidogrel as second antiplatelet (vs. } \\
\text { ticagrelor)** }\end{array}$ & $817(76.3)$ & $143(56.5)$ & $217(78.3)$ & $457(84.5)$ & $<0.001$ \\
\hline GPIlbllla inhibitor administered & $143(13.3)$ & $110(43.3)$ & $20(7.2)$ & $13(2.4)$ & $<0.001$ \\
\hline
\end{tabular}

ting. Complications of $\mathrm{PCl}$ occurred significantly more frequent in case of STEMI (Table 2). A remarkably high incidence of presentation with cardiogenic shock and/ or cardiac arrest was noted: 39 patients $\mathbf{( 7 . 3 \%}$ of the ACS population) suffered from at least one of these conditions.

\section{Mortality after PCI}

The incidence of in-hospital death, and I-, 2- or 3-year cardiovascular mortality was not different between the excluded and included patient groups (Table 3).

Ten of the 536 ACS patients (5.6\%) and none of the 547 CCS patients died in the in-hospital period. As expected, in-hospital mortality was high in patients presenting with cardiogenic shock ( 16 of the 27 patients, 59.3\%) and resuscitated cardiac arrest (II of the 32 patients, $34.4 \%$ ). Conversely, only 10 of the 483 ACS patients presenting without these two conditions
(2.1\%) died during the in-hospital period: 6 of the 210 STEMI- and 4 of the 273 NSTEACS patients $(2.9 \%$ and I.5\%, respectively, $\mathrm{p}=0.28$ ). The in-hospital mortality in the ACS population was $2.8 \%$ in the absence of cardiogenic shock.

After a median follow-up of I 433 days, I 12 patients ( $10.4 \%$ of the whole study population) died of cardiovascular causes. The clinical and procedural variables associated with 3-year cardiovascular mortality are summarized in Table 4. Long-term mortality was also significantly higher in patients who suffered an ACS (Table 3), especially in the case of high-risk presentation. Accordingly, 3-year cardiovascular mortality was as high as $74.1 \%$ and $46.9 \%$ after an ACS complicated by cardiogenic shock or resuscitated cardiac arrest, respectively. If these high-risk cases were excluded, a 3-year post-ACS cardiovascular mortality of II.8\% 


\begin{tabular}{|c|c|c|c|c|c|}
\hline \multirow[b]{2}{*}{ Variable } & \multirow[b]{2}{*}{$\begin{array}{l}\text { Total patients } \\
(\mathbf{N}=1079)\end{array}$} & \multicolumn{4}{|c|}{$\mathrm{PCl}$ indication } \\
\hline & & STEMI $(N=254)$ & NSTEACS $(\mathbf{N}=\mathbf{2 7 8})$ & $\begin{array}{c}\text { CCS } \\
(N=547)\end{array}$ & p \\
\hline Any PCl-related complication & $89(8.2)$ & $50(19.7)$ & $14(5.0)$ & $25(4.6)$ & $<0.001$ \\
\hline Acute vessel closure & $22(2.0)$ & $4(1.6)$ & $6(2.2)$ & $12(2.2)$ & 0.83 \\
\hline Coronary perforation & $10(0.9)$ & $2(0.8)$ & $3(1.1)$ & $5(0.9)$ & 0.93 \\
\hline Angiographic no-reflow phenomenon & $35(3.2)$ & $28(11.0)$ & $2(0.7)$ & $5(0.9)$ & $<0.001$ \\
\hline Heart block requiring emergent pacing & $9(0.8)$ & $7(2.8)$ & $\mathrm{I}(0.4)$ & $\mathrm{I}(0.2)$ & $<0.001$ \\
\hline Resuscitation during $\mathrm{PCl}$ & $13(1.2)$ & $9(3.5)$ & $2(0.7)$ & $2(0.4)$ & $<0.001$ \\
\hline Shock induced by the procedure & $4(0.4)$ & $\mathrm{I}(0.4)$ & $\mathrm{I}(0.4)$ & $2(0.4)$ & 0.99 \\
\hline Stroke & $2(0.2)$ & $0(0.0)$ & $0(0.0)$ & $2(0.4)$ & 0.37 \\
\hline Major bleeding post- $\mathrm{PCl}$ until discharge & $5(0.5)$ & $2(0.8)$ & $\mathrm{I}(0.4)$ & $2(0.4)$ & 0.68 \\
\hline
\end{tabular}

Table 3. Post-PCI mortality rates of the patients included in the PCI Registry

\begin{tabular}{|c|c|c|c|c|c|c|c|}
\hline \multirow[b]{2}{*}{ Mortality } & \multicolumn{3}{|c|}{ Included vs. excluded patients } & \multicolumn{4}{|c|}{ PCI Indication (included patients) } \\
\hline & $\begin{array}{c}\text { Included patients } \\
(\mathrm{N}=1079)\end{array}$ & $\begin{array}{l}\text { Excluded patients } \\
\qquad(\mathrm{N}=\mathbf{8 9})\end{array}$ & $\mathbf{p}$ & $\begin{array}{c}\text { STEMI } \\
(\mathrm{N}=254)\end{array}$ & $\begin{array}{l}\text { NSTEACS } \\
(\mathrm{N}=278)\end{array}$ & $\begin{array}{c}\text { CCS } \\
(N=547)\end{array}$ & P \\
\hline In-hospital mortality & $30(2.8)$ & $3(3.4)$ & 0.74 & $23(9.1)$ & $7(2.5)$ & $0(0.0)$ & $<0.001$ \\
\hline I-year cardiovascular mortality & $58(5.4)$ & $5(5.9)^{*}$ & 0.84 & $34(13.4)$ & $15(5.4)$ & $9(1.6)$ & $<0.001$ \\
\hline 2-year cardiovascular mortality & $8 I(7.5)$ & $6(7.1)^{*}$ & 0.87 & $41(16.1)$ & $20(7.2)$ & $20(3.7)$ & $<0.001$ \\
\hline 3-year cardiovascular mortality & $112(10.4)$ & $6(7.1)^{*}$ & 0.32 & $5 I(20.1)$ & $30(10.8)$ & $31(5.7)$ & $<0.001$ \\
\hline
\end{tabular}

was found. However, this was still significantly higher than the 3-year cardiovascular death rate of $5.7 \%$ observed after elective interventions $(p<0.001)$.

Receiver-operator characteristic curve analysis confirmed the significant association between advanced age and increased mortality after $\mathrm{PCl}$ for an ACS, with a cut-off value of 62 years (area under the curve (AUC): $0.65,95 \% \mathrm{Cl}: 0.60-0.69, \mathrm{p}<0.00 \mathrm{I}$ ). The two Cox proportional hazards regression models predicted 3-year cardiovascular mortality with the following characteristics described by ROC-curve analysis: AUC: 0.86, 95\% Cl: 0.83-0.89, $\mathrm{p}<0.000 \mathrm{I}$ (ACS model), and AUC: $0.7 \mathrm{I}, 95 \% \mathrm{Cl}: 0.67-0.75, \mathrm{p}<0.000 \mathrm{I}$ (CCS model). The independent predictors of long-term cardiovascular mortality after $\mathrm{PCl}$ identified by Cox proportional hazards regression analysis in ACS patients were: the presence of renal dysfunction $(\mathrm{HR}=2.12,95 \% \mathrm{Cl}$ : I.22-3.68, $p<0.01$ ), a depressed left ventricular systolic function (LVEF $\leq 40 \%, H R=3.30,95 \% \mathrm{Cl}$ : I.95-5.59, $P<0.00 \mathrm{I})$, the presentation with cardiogenic shock $(\mathrm{HR}=3.85,95 \% \mathrm{Cl}$ I.9I-7.72, $\mathrm{p}<0.00 \mathrm{I})$ or with resuscitated cardiac arrest $(\mathrm{HR}=3.4,95 \% \mathrm{Cl}$ : I.83-6.43, $\mathrm{P}<0.00 \mathrm{I}$ ). In case of CCS, only two independent pre- dictors of long-term cardiovascular mortality were evidenced: low LVEF (a value of $\leq 40 \%$ was associated with a HR of 3.05, 95\% Cl: I.29-7.24, p = 0.0I) and the history of hemodynamically significant valvular heart disease/prior valvular heart surgery/replacement/intervention $(H R=2.89,95 \% \mathrm{Cl}: 1.20-6.95, p=0.0 I)$. Survival curves according to these independent predictors of long-term mortality are presented in Figure I and Figure 2. In the case of ACS all the independent predictors had an initial significant impact, corresponding to the in-hospital deaths (Figure I); the divergence of the curves is especially striking in case of highrisk clinical presentation, such as cardiogenic shock (Figure Ic) or after resuscitation (Figure Id). Conversely, a more gradual effect of the two independent predictors is observed after elective $\mathrm{PCl}$ (Figure 2).

In conclusion, the main predictors of long-term mortality remained clinical variables: comorbidities and high-risk clinical presentation. None of the angiographic and invasive procedural characteristics or complications had significant, independent impact on the 3-year cardiovascular mortality in this patient population. 


\begin{tabular}{|c|c|c|c|c|c|c|c|c|c|c|c|c|}
\hline \multirow[t]{2}{*}{\begin{tabular}{|l|} 
Variable \\
\end{tabular}} & \multicolumn{3}{|c|}{ Total patients $(\mathrm{N}=1079)$} & \multicolumn{3}{|c|}{ STEMI $(\mathrm{N}=254)$} & \multicolumn{3}{|c|}{ NSTEACS ( $\mathrm{N}=\mathbf{2 7 8 )}$} & \multicolumn{3}{|c|}{$\operatorname{CCS}(\mathrm{N}=547)$} \\
\hline & \begin{tabular}{|c|} 
Deceased \\
$(\mathrm{N}=\mid 12)$
\end{tabular} & $\begin{array}{c}\text { Alive } \\
(\mathrm{N}=967)\end{array}$ & $\mathbf{p}$ & \begin{tabular}{|c|} 
Deceased \\
$(\mathrm{N}=5 \mathrm{I})$
\end{tabular} & $\begin{array}{c}\text { Alive } \\
(\mathrm{N}=203)\end{array}$ & $\mathbf{p}$ & $\begin{array}{l}\text { Deceased } \\
(\mathrm{N}=30)\end{array}$ & $\begin{array}{c}\text { Alive } \\
(\mathrm{N}=248)\end{array}$ & $\mathbf{P}$ & \begin{tabular}{|c|} 
Deceased \\
$(\mathrm{N}=3 \mathrm{I})$
\end{tabular} & \begin{tabular}{|c|} 
Alive \\
$(\mathrm{N}=516)$
\end{tabular} & $\mathbf{p}$ \\
\hline Age (years) & $\begin{array}{c}67.7 \\
(42-89)\end{array}$ & $\begin{array}{c}63.1 \\
(29-89)\end{array}$ & $<0.001$ & $\begin{array}{c}66.1 \\
(42-89)\end{array}$ & $\begin{array}{c}62.0 \\
(31-85)\end{array}$ & 0.001 & $\begin{array}{c}70.2 \\
(48-83)\end{array}$ & $\begin{array}{c}64.4 \\
(29-87)\end{array}$ & 0.001 & $\begin{array}{c}66.1 \\
(49-81)\end{array}$ & $\begin{array}{c}63.1 \\
(37-89)\end{array}$ & 0.07 \\
\hline Female gender & $47(42.0)$ & $282(29.2)$ & $<0.01$ & $25(49.0)$ & $61(30.0)$ & 0.01 & $15(50.0)$ & $88(35.5)$ & 0.12 & $7(22.6)$ & $133(25.8)$ & 0.69 \\
\hline BMI $\left(\mathrm{kg} / \mathrm{m}^{2}\right)$ & $\begin{array}{c}27.8 \\
(15.8-45.3) \\
\end{array}$ & $\begin{array}{c}28.4 \\
(18.6-62.0) \\
\end{array}$ & 0.67 & \begin{tabular}{|c|}
27.7 \\
$(15.8-38.1)$ \\
\end{tabular} & $\begin{array}{c}28.1 \\
(18.6-62.0) \\
\end{array}$ & 0.42 & $\begin{array}{c}27.5 \\
(19.6-44.4)\end{array}$ & $\begin{array}{c}28.4 \\
(19.0-54.2) \\
\end{array}$ & 0.36 & \begin{tabular}{|c|}
29.5 \\
$(23.1-45.3)$ \\
\end{tabular} & \begin{tabular}{|c|}
28.4 \\
$(19.0-50.8)$ \\
\end{tabular} & 0.10 \\
\hline Arterial hypertension & $20(80.2)$ & $92(82.1)$ & 0.63 & $39(76.5)$ & $132(65.0)$ & 0.12 & $6(81.5)$ & $24(80.0)$ & 0.84 & $29(93.5)$ & $442(85.7)$ & 0.21 \\
\hline Diabetes mellitus & $41(36.6)$ & $294(30.4)$ & 0.17 & $12(23.5)$ & $53(26.1)$ & 0.70 & $16(53.3)$ & $79(31.9)$ & 0.01 & $13(41.9)$ & $162(31.4)$ & 0.22 \\
\hline Hypercholesterolemia & $56(50.0)$ & $584(60.4)$ & 0.03 & $21(41.2)$ & $89(43.8)$ & 0.73 & $13(43.3)$ & $152(61.3)$ & 0.05 & $22(66.5)$ & $343(7 \mathrm{I})$ & 0.60 \\
\hline Active smoking & $27(24.1)$ & $209(21.6)$ & 0.54 & $18(35.3)$ & $91(44.8)$ & 0.21 & $3(10.0)$ & $54(21.8)$ & 0.13 & $6(19.4)$ & $64(12.4)$ & 0.26 \\
\hline $\begin{array}{l}\text { History of congestive } \\
\text { heart failure }\end{array}$ & $33(29.5)$ & $118(12.2)$ & $<0.001$ & II (21.6) & $16(7.9)$ & $<0.01$ & II (36.7) & 34 (13.7) & 0.001 & II (35.5) & $68(13.2)$ & $<0.001$ \\
\hline $\begin{array}{l}\text { History of valvular heart } \\
\text { disease }\end{array}$ & $23(20.5)$ & $63(6.5)$ & $<0.001$ & $7(13.7)$ & $10(4.9)$ & 0.02 & $8(26.7)$ & $20(8.1)$ & 0.001 & $8(28.5)$ & $33(6.4)$ & $<0.001$ \\
\hline \begin{tabular}{|l|}
$\begin{array}{l}\text { History of peripheral } \\
\text { arterial disease }\end{array}$ \\
\end{tabular} & $19(17.0)$ & $127(13.1)$ & 0.26 & $5(9.8)$ & $8(3.9)$ & 0.09 & $7(23.3)$ & $28(11.3)$ & 0.06 & $7(22.6)$ & 91 (I7.6) & 0.48 \\
\hline History of stroke & $59(6.1)$ & $9(8.0)$ & 0.42 & $2(3.9)$ & $15(7.4)$ & 0.37 & $6(20.0)$ & $18(7.3)$ & 0.01 & $I(3.2)$ & $26(5.0)$ & 0.65 \\
\hline $\begin{array}{l}\text { Previous myocardial } \\
\text { infarction }\end{array}$ & $35(31.2)$ & $273(28.2)$ & 0.50 & $9(17.6)$ & $26(12.8)$ & 0.37 & $10(33.3)$ & $64(25.8)$ & 0.37 & $16(51.6)$ & $183(35.5)$ & 0.06 \\
\hline Previous $\mathrm{PCl}$ & $24(21.4)$ & $268(27.7)$ & 0.15 & $6(11.8)$ & $14(6.9)$ & 0.24 & $7(23.3)$ & $60(24.2)$ & 0.91 & II (35.5) & $194(37.6)$ & 0.81 \\
\hline Previous CABG & $2(1.8)$ & $43(4.4)$ & 0.18 & $0(0.0)$ & $2(1.0)$ & 0.47 & $0(0.0)$ & II (4.4) & 0.24 & $2(6.5)$ & $30(5.8)$ & 0.88 \\
\hline $\mathrm{CrCl}(\mathrm{ml} / \mathrm{min})$ & $\begin{array}{c}79.2 \\
(7.0-175.6) \\
\end{array}$ & \begin{tabular}{|c|}
$107.4(7.6-$ \\
$711.8)$ \\
\end{tabular} & $<0.001$ & $\begin{array}{c}59.0(8.2- \\
154.3)\end{array}$ & $\begin{array}{c}111.4 \\
(15.5-263.6) \\
\end{array}$ & $<0.001$ & $\begin{array}{c}71.1 \\
(34.8-124.6) \\
\end{array}$ & $\begin{array}{c}103.6 \\
(26.0-285.4) \\
\end{array}$ & $<0.001$ & $\begin{array}{c}112.5 \\
(7.0-175.6) \\
\end{array}$ & \begin{tabular}{|c|}
106.8 \\
$(7.6-7 \mid 1.8)$ \\
\end{tabular} & 0.71 \\
\hline $\begin{array}{l}\text { Left ventricular ejection } \\
\text { fraction }(\%)\end{array}$ & $40(10-65)$ & $50(20-80)$ & $<0.001$ & $35(10-60)$ & $45(25-60)$ & $<0.001$ & $45(20-60)$ & $53(20-65)$ & $<0.001$ & $45(25-65)$ & $55(20-80)$ & $<0.01$ \\
\hline \begin{tabular}{|l|} 
Presentation with \\
cardiogenic shock
\end{tabular} & $20(17.9)$ & $7(0.7)$ & $<0.001$ & $18(35.3)$ & $7(3.4)$ & $<0.001$ & $2(6.7)$ & $0(0.0)$ & $<0.001$ & 0 & 0 & - \\
\hline Cardiac arrest before $\mathrm{PCl}$ & $15(13.4)$ & $17(1.8)$ & $<0.001$ & $12(23.5)$ & $16(7.9)$ & 0.001 & $3(10.0)$ & $\mathrm{I}(0.4)$ & $<0.001$ & 0 & 0 & - \\
\hline Radial arterial access site & $55(49.1)$ & $653(67.5)$ & 0.001 & $16(31.4)$ & $120(59.1)$ & $<0.001$ & $17(56.7)$ & $165(66.5)$ & 0.28 & $22(71.0)$ & $368(71.3)$ & 0.96 \\
\hline Three-vessel disease & 49 (43.7) & $340(35.2)$ & 0.07 & $20(39.2)$ & $57(28.1)$ & 0.12 & $13(43.3)$ & $93(37.5)$ & 0.53 & $16(51.6)$ & $190(36.8)$ & 0.09 \\
\hline Left main stem disease & $19(17.0)$ & $77(8.0)$ & 0.001 & $8(15.7)$ & $9(4.4)$ & $<0.01$ & $8(2.7)$ & $23(9.3)$ & $<0.01$ & $3(9.7)$ & $45(8.7)$ & 0.85 \\
\hline TIMI flow <2 post-PCl & $8(7.1)$ & $27(2.8)$ & 0.01 & $4(7.8)$ & $2(1.0)$ & $<0.01$ & $3(10.0)$ & $8(3.2)$ & 0.07 & $I(3.2)$ & $17(3.3)$ & 0.98 \\
\hline $\begin{array}{l}\mathrm{PCl} \text { without stent } \\
\text { implantation }\end{array}$ & $17(15.2)$ & $64(6.6)$ & 0.001 & $9(17.6)$ & $10(4.9)$ & $<0.01$ & $7(23.3)$ & $19(7.7)$ & $<0.01$ & I (3.2) & $35(6.8)$ & 0.43 \\
\hline $\begin{array}{l}\text { Number of implanted } \\
\text { stents }>1\end{array}$ & $35(31.2)$ & $293(30.3)$ & 0.83 & $10(19.6)$ & $48(23.6)$ & 0.54 & $12(40.0)$ & $85(34.3)$ & 0.53 & $13(41.9)$ & $160(31.0)$ & 0.20 \\
\hline $\begin{array}{l}\text { Only drug-eluting stents } \\
\text { implanted* }\end{array}$ & $83(87.4)$ & $851(94.2)$ & $<0.01$ & $33(78.6)$ & $176(91.2)$ & 0.01 & $20(87.0)$ & $221(96.5)$ & 0.03 & $30(100.0)$ & $454(94.4)$ & 0.18 \\
\hline Dual antiplatelet therapy & $104(92.9)$ & $927(95.9)$ & 0.14 & $49(96.1)$ & $199(98.0)$ & 0.41 & $28(93.3)$ & $239(96.4)$ & 0.42 & $27(87.1)$ & $489(94.8)$ & 0.07 \\
\hline $\begin{array}{l}\text { Clopidogrel as second } \\
\text { antiplatelet (vs. } \\
\text { ticagrelor)** }\end{array}$ & $85(77.3)$ & $732(76.2)$ & 0.79 & $31(62.0)$ & $112(55.2)$ & 0.38 & $26(89.7)$ & 191 (77.0) & 0.11 & $28(90.3)$ & $429(84.1)$ & 0.35 \\
\hline $\begin{array}{l}\text { GPIllbllla inhibitor } \\
\text { administered }\end{array}$ & $25(22.3)$ & $118(12.2)$ & $<0.01$ & $22(43.1)$ & $88(43.3)$ & 0.97 & $3(10.0)$ & $17(6.9)$ & 0.52 & $0(0.0)$ & $13(2.5)$ & 0.37 \\
\hline $\begin{array}{l}\text { Any PCl-related } \\
\text { complication }\end{array}$ & $23(20.5)$ & $66(6.8)$ & $<0.001$ & $19(37.3)$ & 31 (15.3) & $<0.001$ & $3(10.0)$ & II (4.4) & 0.18 & I (3.2) & $24(4.7)$ & 0.71 \\
\hline tegorical data are & as freq & $=5$ (I) & & (T. & 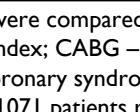 & & & & & & & \\
\hline
\end{tabular}

\section{DISCUSSION}

To the best of our knowledge, the present work is the first one evaluating the long-term post- $\mathrm{PCl}$ mortality and its predictors in an all-comers population in Romania. Patients were prospectively included in the institutional PCl Registry, and a complete 3-year follow-up was accomplished regarding cardiovascular mortality in the selected population. Nevertheless, the results reflect the remarkable technical advancement emerged worldwide in the field of percutaneous coronary revascularization in the past few decades (high procedural success rate, the more frequent use of radial arterial approach and drug-eluting stents). 


\section{Cardiovascular death rate}

The average in-hospital and 3-year mortality after $\mathrm{PCl}$ in different recent large registry studies ranges between $0.65-2.60 \%{ }^{25-27}$ and $5.6-9.8 \%{ }^{28-30}$, respectively. However, very high risk patients (e.g. with cardiogenic shock) were excluded ${ }^{25}$ or represented substantially less ${ }^{26-30}$ in these works, partially explaining the higher mortality rates observed in our cohort. The presentation with cardiogenic shock in the analyzed ACS population (i.e. $5.1 \%$, Table I) was higher than the incidence of this complication in the ACS population of the Coronary Angiography and $\mathrm{PCl}$ registry of the German Cardiac Society (3.8\%) ${ }^{27}$. The in-hospital mortality rates of these two populations are quite similar in the absence of cardiogenic shock: $2.8 \%$ in our cohort and $2.9 \%$ in the German ACS population ${ }^{27}$. However, the presentation with cardiogenic shock was associated with an undoubtedly higher in-hospital mortality rate in our ACS population (59.3\% vs. $42.2 \%$ in the German ACS registry). These observations might contribute to the explanation of the higher CAD mortality rates observed in Romania ${ }^{2}$, and emphasizes the necessity of patient information and education, as well as the need for the continuous development of the existing intensive care facilities in our country.

\section{Predictors of long-term mortality}

While in the case of ACS both clinical and procedural factors were identified as univariate predictors of longterm post-PCl mortality, this outcome was associated with only clinical variables after elective procedures (Table 4). However, multivariate analysis evidenced as independent predictors of long-term cardiovascular mortality only some of the clinical characteristics even in ACS patients.

Not surprisingly, the history of significant valvular heart disease was associated with 3-year cardiovascular mortality in ACS and CCS patients. However, this association was an independent one only in the case of elective interventions in our patient population. Both conditions: CAD and valvular heart disease could lead to cardiovascular death. Moreover, aortic stenosis ${ }^{31}$ and mitral regurgitation ${ }^{32}$ were both identified previously as independent predictors of mortality in different subsets of CAD patients.

Renal dysfunction, a well-known predictor of mortality in CAD patients ${ }^{33-35}$ was also an independent predictor of long term mortality after $\mathrm{PCl}$ for ACS . In addition to the possible acute kidney injury related to the $\mathrm{PCl}$ procedure itself $\mathrm{f}^{36}$, renal dysfunction is associated with the higher occurrence of other procedu- re- or disease-related complications, such as the noreflow phenomenon ${ }^{37}$ or atrial fibrillation ${ }^{38}$ in case of percutaneous coronary revascularization for STEMI.

The depressed systolic function of the left ventricle was the single independent predictor of long-term cardiovascular mortality after both urgent and elective $\mathrm{PCl}$. This negative effect of left ventricular dysfunction on post-PCl mortality is well-known ${ }^{26,39}$; left ventricular ejection fraction is incorporated in many prognostic scores currently used for risk stratification in CAD patients ${ }^{40-42}$.

Renal dysfunction and poor left ventricular function had a similar effect on the followed clinical outcome: both conditions were associated with an abrupt increase in mortality during hospital admission, followed by a more gradual, but continuous divergence of the Kaplan-Meier curves in case of ACS (Figure la and b). Contrary to this, other independent predictors of long-term mortality after an ACS, such as presentation with cardiogenic shock or resuscitated cardiac arrest had mainly an acute initial impact, remarkably increasing in-hospital mortality (Figure Ic and d).

The mortality of resuscitated patients and those with cardiogenic shock is disappointingly high, even in the current era of emergent revascularization in $\mathrm{PCl}$ centers with $24 / 7$ availability ${ }^{7,27,43}$. Although there is a continuous effort to reduce this high mortality, including the development of dedicated "regionalized cardiogenic shock systems" ters" 44 equipped with extracorporeal life support and ventricular assist devices, these measures are mainly unavailable in our country ${ }^{7}$. However, as cardiogenic shock complicating acute coronary syndromes is one of the main determinants of post- $\mathrm{PCl}$ long-term mortality ${ }^{7,27}$ and given its early major impact on survival, these measures applied as early as possible in cardiogenic shock patients might represent a possible way to lower further the long-term mortality rate of CAD in Romania.

\section{Study limitations}

The major limitation of our study is the reduced number of the included patients, all of them recruited from a single center. In consequence, our results should be interpreted with some caution regarding the possibility of insufficient statistical power. However, we tried to reduce this limitation by extensive data collection and excluding as few patients as possible. The reader cannot consider these results as representative for the whole country; however, results from large multicenter registries are still waited for this purpose. The 
current research represents preliminary findings from an on-going $\mathrm{PCl}$ registry. The future results of this project probably will add further details regarding the evolution of the disease causing most of the deaths in Romania.

\section{CONCLUSIONS}

Clinical variables (poor left ventricular function, renal dysfunction, presentation with cardiogenic shock or cardiac arrest in the case of ACS; low LVEF and the history of valvular heart disease in the case of CCS), but no procedural factors were the main predictors of 3-year cardiovascular mortality after $\mathrm{PCl}$ in this allcomers population.

\section{Conflict of interest: none declared.}

Acknowledgements: The authors would like to thank all the resident colleagues who continuously introduce the data regarding $\mathrm{PCls}$ performed in the Emergency Institute for Cardiovascular Diseases and Transplantation since 2016. We also would like to thank Mr. loan Matei, for his help in achieving longterm mortality data.

Funding: This study was funded by the Romanian Academy of Medical Sciences and European Regional Development Fund, within the framework of a twophased research project entitled: „Dezvoltarea infrastructurii publice de cercetare, dezvoltare și crearea de noi infrastructuri": Phase I: SMIS 56377, Funding contract 670/30.01.2015, and Phase 2: MySMIS 107I24, Funding contract 2/Axa I/3I.07.20I7.

\section{References}

I. Simionescu M, Bilan S, Gavurova B, Bordea EN. Health Policies in Romania to Reduce the Mortality Caused by Cardiovascular Diseases. Int J Environ Res Public Health 2019;16: 3080.

2. Cardiovascular diseases statistics. Available on-line at: https:// ec.europa.eu/eurostat/statistics-explained/pdfscache/37359.pdf. Accessed on 07 August 2020).

3. Serban RC, Hadadi L, Sus I, Lakatos EK, Demjen Z, Scridon A. Impact of chronic obstructive pulmonary disease on in-hospital morbidity and mortality in patients with ST-segment elevation myocardial infarction treated by primary percutaneous coronary intervention. Int J Cardiol 2017;243: 437-42.

4. Bordejevic DA, Caruntu F, Mornos C, Olariu I, Petrescu L, Tomescu MC, Citu A, Mavrea A, Pescariu S. Prognostic impact of blood pressure and heart rate at admission on in-hospital mortality after primary percutaneous intervention for acute myocardial infarction with ST-segment elevation in western Romania. Ther Clin Risk Manag 2017; I3: 106I-8.

5. Benedek I, Gyongyosi M, Benedek T. A prospective regional registry of ST-elevation myocardial infarction in Central Romania: impact of the Stent for Life Initiative recommendations on patient outcomes. Am Heart J 20I3;166: 457-65.

6. Barcan A, Suciu Z, Benedek I. Response to Fragmented QRS Complex Predicts Contrast-Induced Nephropathy and In-Hospital Mortality After Primary Percutaneous Coronary Intervention in Patients
With ST-Segment Elevation Myocardial Infarction. Clin Cardiol 2017;40: II74-5

7. Burlacu A, Tinica G, Nedelciuc I, Simion P, Artene B, Covic A. Strategies to Lower In-Hospital Mortality in STEMI Patients with Primary PCl: Analysing Two Years Data from a High-Volume Interventional Centre. J Interv Cardiol 2019;2019: 3402081.

8. Cenko E, Ricci B, Kedev S, Vasiljevic Z, Dorobantu M, Gustiene O, Kneževic B, Milicic D, Dilic M, Trninic D, Smith F, Manfrini O, Badimon L, Bugiardini R. Reperfusion therapy for ST-elevation acute myocardial infarction in Eastern Europe: the ISACS-TC registry. Eur Heart J Qual Care Clin Outcomes 2016;2: 45-5I.

9. Tatu-Chitoiu G, Arafat R, Deleanu D, Vinereanu D, Udroiu C, Petris A. Impact of the Romanian national programme for interventional therapy in ST-elevation myocardial infarction. Eurolntervention 20I2;8 Suppl P: PI26-32.

10. Cretu DE, Udroiu CA, Stoicescu Cl, Tatu-Chitoiu G, Vinereanu D. Predictors of in-Hospital Mortality of ST-Segment Elevation Myocardial Infarction Patients Undergoing Interventional Treatment. An Analysis of Data from the RO-STEMI Registry. Maedica 2015;10: 295-303.

II. Ceamburu A, Serban RC, Sus I, Lakatos EK, Demjen Z, Fisca PC, Hadadi L, Somkereki C, Scridon A. Gender equality applies (partially) to ST-segment elevation myocardial infarction too. Rom J Cardiol 2019;29: 218-24.

12. Deak E, Brumboiu MI, Tigan S, Bocsan IS. Observatii epidemiologice legate de supravietuirea in primul an dupa infarct miocardic acut $\mathrm{Cu}$ supradenivelare de segment ST (STEMI) tratat prin angioplastie primara. Rev Med Chir Soc Med Nat lasi 20I I; I I5: 905-I3.

13. Olinic D, Desveaux B, Quillet L, Maillard L, Paemelaere JM, Pacouret G, Charbonnier B, Raynaud P. Primary coronary angioplasty in acute myocardial infarction excluded from thrombolysis: in-hospital and mid-term results. Rom I Intern Med 1998;36: 3-I5.

14. Homorodean C, lancu AC, Dregoesc IM, Spinu M, Ober MC, Tataru $D$, Leucuța D, Olinic M, Olinic DM. Renal Failure Impact on the Outcomes of ST-Segment Elevation Myocardial Infarction Patients Due to a Left Main Coronary Culprit Lesion Treated Using a Primary Percutaneous Coronary Intervention. J Clin Med 2019;8: 565.

15. Homorodean C, lancu AC, Leucuta D, Balanescu S, Dregoesc IM, Spinu M, Ober M, Tãtaru D, Olinic M, Bindea D, Olinic D. New Predictors of Early and Late Outcomes after Primary Percutaneous Coronary Intervention in Patients with ST-Segment Elevation Myocardial Infarction and Unprotected Left Main Coronary Artery Culprit Lesion. J Interv Cardiol 2019;2019: 8238972.

16. Flynn MR, Barrett C, Cosio FG, Gitt AK, Wallentin L, Kearney P, Lonergan M, Shelley E, Simoons ML. The Cardiology Audit and Registration Data Standards (CARDS), European data standards for clinical cardiology practice. Eur Heart J 2005;26: 308-13.

17. Thygesen K, Alpert JS, Jaffe AS, Simoons ML, Chaitman BR, White HD, Joint ESC/ACCF/AHA/WHF Task Force for Universal Definition of Myocardial Infarction. Third universal definition of myocardial infarction. Eur Heart J 20I2;33: 255I-67.

18. Roffi M, Patrono C, Collet JP, Mueller C, Valgimigli M, Andreotti F, Bax JJ, Borger MA, Brotons C, Chew DP, Gencer B, Hasenfuss G, Kjeldsen K, Lancellotti P, Landmesser U, Mehilli J, Mukherjee D, Storey RF, Windecker S, ESC Scientific Document Group. 2015 ESC Guidelines for the management of acute coronary syndromes in patients presenting without persistent ST-segment elevation: Task Force for the Management of Acute Coronary Syndromes in $\mathrm{Pa}$ tients Presenting without Persistent ST-Segment Elevation of the European Society of Cardiology (ESC). Eur Heart J 2016;37: 267-3I5.

19. Knuuti J, Wijns W, Saraste A, Capodanno D, Barbato E, Funck-Brentano C, Prescott E, Storey RF, Deaton C, Cuisset T, Agewall S, Dickstein K, Edvardsen T, Escaned J, Gersh BJ, Svitil P, Gilard M, Hasda D, Hatala R, Mahfoud F, Masip J, Muneretto C, Valgimigli M, Achenbach S, Bax JJ, ESC Scientific Document Group. 2019 ESC Guidelines for the diagnosis and management of chronic coronary syndromes. Eur Heart J 2020;4I: 407-77.

20. Authors/Task Force $m$, Windecker $S$, Kolh P, Alfonso F, Collet JP, Cremer J, Falk V, Filippatos G, Hamm C, Head SJ, Jüni P, Kappetein 
AP, Kastrati A, Knuuti J, Landmesser U, Laufer G, Neumann FJ, Richter DJ, Schauerte P,Sousa Uva M, Stefanini GG, Taggart DP, Torracca L, Valgimigli M, Wijns W, Witkowski A. 2014 ESC/EACTS Guidelines on myocardial revascularization: The Task Force on Myocardial Revascularization of the European Society of Cardiology (ESC) and the European Association for Cardio-Thoracic Surgery (EACTS)Developed with the special contribution of the European Association of Percutaneous Cardiovascular Interventions (EAPCI). Eur Heart J 2014;35: 254I-619.

21. Levey AS, Eckardt KU, Tsukamoto Y, Levin A, Coresh J, Rossert J, De Zeeuw D, Hostetter TH, Lameire N, Eknoyan G. Definition and classification of chronic kidney disease: a position statement from Kidney Disease: Improving Global Outcomes (KDIGO). Kidney Int 2005;67: 2089-100.

22. Cockcroft DW, Gault MH. Prediction of creatinine clearance from serum creatinine. Nephron 1976; 16: 31-4I.

23. Eeckhout E, Kern MJ. The coronary no-reflow phenomenon: a review of mechanisms and therapies. European heart journal. 200I May;22(9):729-39.

24. Lang RM, Badano LP, Mor-Avi V, Afilalo J, Armstrong A, Ernande L, Flachskampf FA, Foster E, Goldstein SA, Kuznetsova T, Lancellotti P, Muraru D, Picard MH, Rietzschel ER, Rudski L, Spencer KT, Tsang $\mathrm{W}$, Voigt JU. Recommendations for cardiac chamber quantification by echocardiography in adults: an update from the American Society of Echocardiography and the European Association of Cardiovascular Imaging. J Am Soc Echocardiogr 20I5;28: I-39 el 4.

25. Chhatriwalla AK, Amin AP, Kennedy KF, House JA, Cohen DJ, Rao SV, Messenger JC, Marso SP, National Cardiovascular Data Registry. Association between bleeding events and in-hospital mortality after percutaneous coronary intervention. JAMA 2013;309: 1022-9.

26. Lodi-Junqueira L, da Silva JL, Ferreira LR, Goncalves HL, Athayde GR, Gomes TO, Borges JC, Nascimento BR, Lemos PA, Ribeiro ALP. In-hospital mortality risk prediction after percutaneous coronary interventions: Validating and updating the Toronto score in Brazil. Catheter Cardiovasc Interv 2015;86: E239-46.

27. Heer T, Hochadel M, Schmidt K, Mehilli J, Zahn R, Kuck KH, Hamm C, Böhm M, Ertl G, Hoffmeister HM, Sack S, Senges J, Massberg S, Gitt AK, Zeymer U. Sex Differences in Percutaneous Coronary Intervention-Insights From the Coronary Angiography and PCI Registry of the German Society of Cardiology. J Am Heart Assoc 2017;6: e004972.

28. Natsuaki M, Morimoto T, Furukawa Y, Nakagawa Y, Kadota K, Iwabuchi M, Shizuta S, Shiomi H, Kimura T. Comparison of 3-year clinical outcomes after transradial versus transfemoral percutaneous coronary intervention. Cardiovasc Interv Ther 2012;27: 84-92.

29. Kovacic JC, Mehran R, Karajgikar R, Baber U, Suleman J, Kim MC, Krishnan P, Dangas G, Sharma SK, Kini A. Female gender and mortality after percutaneous coronary intervention: results from a large registry. Catheter Cardiovasc Interv 20I2;80: 5I4-2I.

30. Wu C, Camacho FT, King SB, 3rd, Walford G, Holmes DR, Jr., Stamato NJ, Berger PB, Sharma S, Curtis JP, Venditti FJ, Jacobs AK, Hannan EL. Risk stratification for long-term mortality after percutaneous coronary intervention. Circ Cardiovasc Interv 2014;7: 80-7.

3I. Kuchulakanti P, Rha SW, Satler LF, Suddath WO, Pichard AD, Kent KM, Weissman NJ, Cheneau E, Pakala R, Canos DA, Pinnow EE, Waksman. Safety of percutaneous coronary intervention alone in symptomatic patients with moderate and severe valvular aortic stenosis and coexisting coronary artery disease: analysis of results in 56 patients. J Invasive Cardiol 2004;16: 688-91.

32. Li S, Barywani S, Fu M. Prognostic significance of mitral regurgitation in long-term all-cause mortality in patients aged $>/=80$ years with acute coronary syndrome. Int J Cardiol 2014; I 76: 340-5.

33. Gupta T, Paul N, Kolte D, Harikrishnan P, Khera S, Aronow WS, Mujib M, Palaniswamy C, Sule S, Jain D, Ahmed A, Cooper HA, Frish- man WH, Bhatt DL, Fonarow GC, Panza JA. Association of chronic renal insufficiency with in-hospital outcomes after percutaneous coronary intervention. J Am Heart Assoc 2015;4: e002069.

34. Hadadi L, Somkereki C, Dobreanu, D. Renal dysfunction at hospital admission, high complexity of coronary artery disease and short term prognosis in acute ST-segment elevation myocardial infarction. Rev Rom Med Lab 201 2;20: 363-9.

35. Choong Hou K, Sin Yoong Kong K, Kee Yi Shern T, Tan Wei Chieh J. Impact of Chronic Kidney Insufficiency on Cardiovascular Outcomes in Patients that Undergo Coronary Revascularization: A Historical Review. ASEAN Heart J 2016;24: 8.

36. Brown JR, Solomon RJ, Robey RB, Plomondon ME, Maddox TM, Marshall EJ, Nichols EL, Matheny ME, Tsai TT, Rumsfeld JS, Lee RE, Sarnak MJ. Chronic Kidney Disease Progression and Cardiovascular Outcomes Following Cardiac Catheterization-A Population-Controlled Study. J Am Heart Assoc 2016;5: e0038I2.

37. Hadadi L, Serban RC, Scridon A, Sus I, Lakatos EK, Demjen Z, Dobreanu $D$. Clinical risk scores predict procedural complications of primary percutaneous coronary intervention. Anatol J Cardiol 2017;17: 276-84

38. Serban RC, Sus I, Lakatos EK, Demjen Z, Ceamburu A, Fisca PC, Somekreki C, Hadadi L, Scridon A. Chronic kidney disease predicts atrial fibrillation in patients with ST-segment elevation myocardial infarction treated by primary percutaneous coronary intervention. Acta Cardiol 2019;74: 472-9.

39. Rasoul S, Ottervanger JP, de Boer MJ, Dambrink JH, Hoorntje JC, Marcel Gosselink AT, Zijlstra F, Suryapranata H, van't Hof AWJ, Zwolle Myocardial Infarction Study Group. Predictors of 30-day and I-year mortality after primary percutaneous coronary intervention for ST-elevation myocardial infarction. Coron Artery Dis 2009;20: 4I5-2I.

40. Nashef SA, Roques F, Michel P, Gauducheau E, Lemeshow S, Salamon R. European system for cardiac operative risk evaluation (EuroSCORE). Eur J Cardiothorac Surg 1999; 16: 9-13.

41. Farooq V, van Klaveren D, Steyerberg EW, Meliga E, Vergouwe $Y$, Chieffo A, Kappetein AP, Colombo A, Holmes DR Jr, Mack M, Feldman T, Morice MC, Ståhle E, Onuma Y, Morel M, Garcia-Garcia HM, van Es GA, Dawkins KD, Mohr FW, Serruys PW. Anatomical and clinical characteristics to guide decision making between coronary artery bypass surgery and percutaneous coronary intervention for individual patients: development and validation of SYNTAX score II. Lancet 2013;38I: 639-50.

42. Peterson ED, Dai D, DeLong ER, Brennan JM, Singh M, Rao SV, Shaw RE, Roe MT, Ho KKL, Klein LW, Krone RJ, Weintraub WS, Brindis RG, Rumsfeld JS, Spertus JA, NCDR Registry Participants. Contemporary mortality risk prediction for percutaneous coronary intervention: results from 588,398 procedures in the National Cardiovascular Data Registry. JACC 2010;55: 1923-32.

43. Garot $P$, Lefevre $T$, Eltchanin off $H$, Morice MC, Tamion F, Abry B, Lesault PF, Le Tarnec JY, Pouges C, Margenet A, Monchi M, Laurent I, Dumas P, Garot J, Louvard Y. Six-month outcome of emergency percutaneous coronary intervention in resuscitated patients after cardiac arrest complicating ST-elevation myocardial infarction. Circulation 2007; | | 5: |354-62.

44. van Diepen S, Katz JN, Albert NM, Henry TD, Jacobs AK, Kapur NK, Kilic A, Menon V, Ohman EM, Sweitzer NK, Thiele H, Washam JB, Cohen MG, American Heart Association Council on Clinical Cardiology; Council on Cardiovascular and Stroke Nursing; Council on Quality of Care and Outcomes Research; and Mission: Lifeline. Contemporary Management of Cardiogenic Shock: A Scientific Statement From the American Heart Association. Circulation 2017;136: e232-e68. 